\title{
A Framework for Situated Multiagent Systems
}

\author{
Danny Weyns and Tom Holvoet \\ DistriNet, Katholieke Universiteit Leuven \\ Celestijnenlaan 200 A, B-3001 Leuven, Belgium \\ \{danny.weyns, tom.holvoet\}@cs.kuleuven.be
}

\begin{abstract}
In this paper, we present an object-oriented framework for situated multiagent systems. The framework integrates various mechanisms for adaptivity we have developed and applied in our research, including selective perception, protocol-based communication, behavior-based decision making with roles and situated commitments, and laws that mediate the activities of agents in the environment. The framework provides a reusable design asset that facilitates the development of new multiagent system applications that share the common base more reliable and cost efficiently. We give an overview of the framework, and we zoom in on two particular features: decision making with a free-flow tree and support for simultaneous actions. Finally, we show how the framework is applied to an experimental robot application.
\end{abstract}

\section{Introduction}

In our research, we study the engineering of software systems with two particular characteristics: (1) the systems are subject to highly dynamic and changing operating conditions such as dynamically changing workloads and variations in the availability of resources, and (2) activity in the systems is inherently localized, i.e. global control is difficult to achieve or even infeasible. Example domains are peer-to-peer file sharing systems, wireless sensor networks, and automated traffic and transportation systems.

To deal with the dynamics and the inherent locality of activity, we apply the paradigm of situated multiagent systems. During the last five years, we have developed several mechanisms of adaptivity for situated multiagent systems, including selective perception [33], protocol-based communication [32], behavior-based decision making with roles and situated commitments [22], and laws that mediate the activities of agents in the environment [28]. We have applied these mechanisms in various applications, ranging from experimental simulations [24] and prototypical robot applications [31] up to an industrial transportation system for automatic guided vehicles [30].

Based on these experiences, we have developed an object-oriented framework for situated multiagent systems. The framework aims to support the development of experimental applications with characteristics similar to the systems we target in our research. Particular motivations for the framework development are: (1) it integrates the various mechanisms for adaptivity in an abstract design, (2) it provides a reusable design asset that allows developers to derive new situated multiagent systems that share the common base more reliable and cost efficiently, (3) it provides a tool for investigating, experimenting and evaluating new concepts and mechanisms of situated multiagent systems.

R. Choren et al. (Eds.): SELMAS 2006, LNCS 4408, pp. 204231, 2007.

(C) Springer-Verlag Berlin Heidelberg 2007 
In this paper, we give an overview of the framework for situated multiagent systems. We describe the core of the framework (frozen spot) that is common to all applications derived from the framework, and the hot-spots that represent the variable parts which allow a framework to be adapted to a particular application [11]. We provide a more detailed explanation of two particular features: decision making with a free-flow tree and support for simultaneous actions.

The framework allows the development of situated agent systems with a software environment as well as systems with a physical environment. It provides no support for distribution of a software environment. The framework can be classified in the middle between whitebox and blackbox [11]. Some parts of the framework core are completely hidden for the application developer, an example is the synchronization of simultaneous actions. Other parts however, require knowledge of the internals of the framework. The framework is implemented in Java 1.5 and is available for download [1]. [34] provides a detailed documentation of the framework in the form of a cookbook [6].

Overview. The paper is structured as follows. We start with a brief introduction of the Packet-World that we will use to illustrate the explanation of the framework. Section 3 then presents the main packages of the framework and discusses the two basic parts of the framework: agent and application environment. Section 4 zooms in on decision making with a free-flow tree, and Sect.5 explains how simultaneous actions are supported in the framework. Section 6 explains failure treatment in the framework. In Sect. 7] we show how the framework is applied to an experimental robot application. Section 8 points out the typical differences between the framework and other multiagent system development frameworks. Finally, Sect.9 draws conclusions.

\section{The Packet-World}

Before we start with explaining the framework, we briefly introduce the Packet-World that we will use as an illustrative case throughout this paper. The basic setup of the Packet-World consists of a number of differently colored packets that are scattered over a rectangular grid. Agents that live in this virtual world have to collect these packets and bring them to the correspondingly colored destination. Figure 1(a) shows an example of a Packet-World of size 10x10 with 8 agents (symbolized by the little fellows).

Colored rectangles symbolize packets that can be manipulated by the agents and circles symbolize destinations. The battery symbol at the bottom row of the grid symbolizes a battery charger.

In the Packet-World, agents can interact with the environment in a number of ways. Agents can make a step to a free neighboring cell. If an agent is not carrying any packet, it can pick up a packet from one of its neighboring cells. An agent can put down a packet it carries at one of the free neighboring cells, or of course at the destination point of that particular packet. Agents can also pass packets to neighboring agents forming a chain. Such a chain enables agents to deliver packets more efficiently, e.g. in the situation of Fig. 1(a) agent 1 can pass a packet to agent 8 that can deliver the packet directly at the destination. Finally, if there is no sensible action for an agent to perform, it may wait for a while and do nothing. Besides acting in the environment, agents can also send messages to each other. In particular, agents can request each other for information 


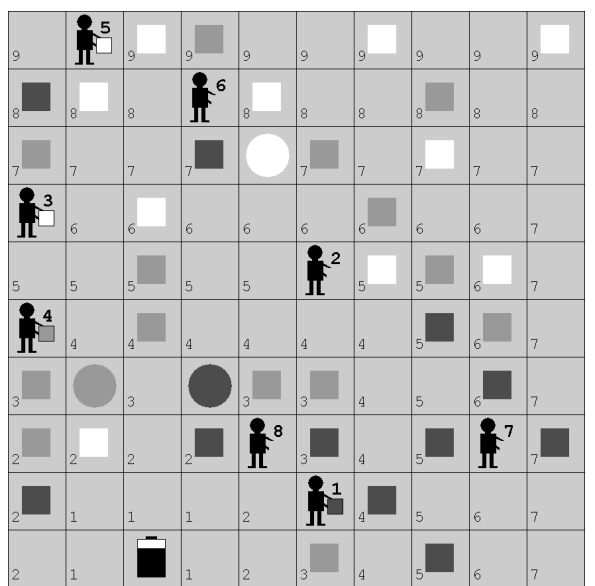

(a) A Packet-World of 10x10

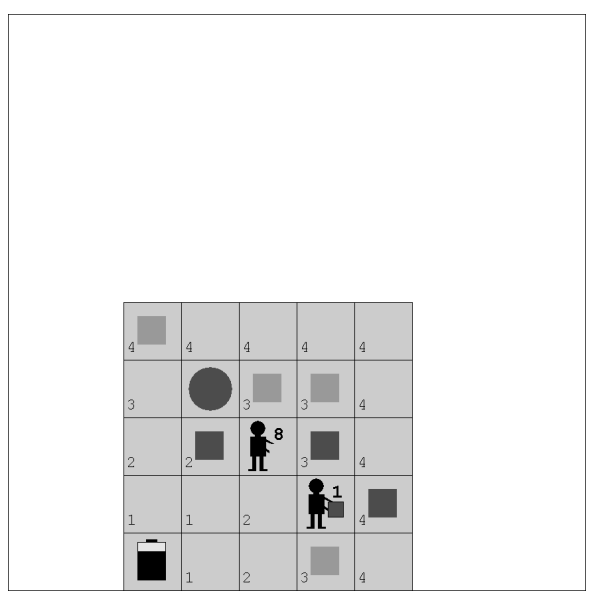

(b) Local view of agent 8

Fig. 1. Example of the Packet-World

about packets or destinations and set up collaborations. The goal of the agents is to perform their job efficiently, i.e. clear up the packets with a minimum number of steps, packet manipulations, and message exchanges.

Agents in the Packet-World can access the environment only to a limited extent. Agents can only manipulate objects in their direct vicinity. The sensing-range of the world expresses how far, i.e. how many squares, an agent can perceive its neighborhood. Figure (1(b) illustrates the limited view of agent 8 , in this example the sensing-range is 2. Similarly, the communication-range determines the scope within which agents can communicate with one another.

Performing actions requires energy. Therefore agents are equipped with a battery. The energy level of the battery is of vital importance to the agents. The battery can be charged at the battery charger. The charger emits a gradient field, i.e. a force field that is spread in the environment and that can be sensed by the agents. The intensity of the field increases further away from the charger. To navigate towards a battery charger, the agents follow the gradient of the field in the direction of decreasing values. The value of the gradient field is indicated by a small number in the bottom left corner of each cell.

In addition to the basic setup, the Packet-World also supports indirect coordination among agents via markers in the environment. A typical example are digital pheromones that agents use to form paths between a cluster of packets and the corresponding destination. For more details about the Packet-World we refer to [24].

\section{General Overview of the Framework}

Figure 2 shows a general overview of the packages of the framework for situated multiagent systems.

The Agent and Application Environment packages encapsulate the core of the framework and provide factories to create agents and the application environment. 


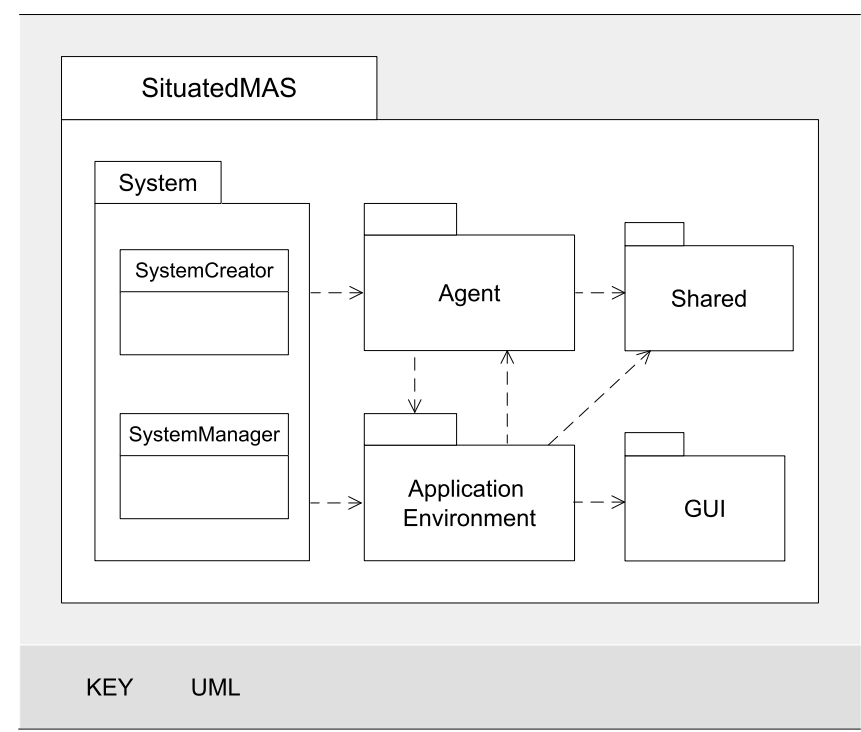

Fig. 2. General overview of the framework

The Shared package encapsulates helper classes for Agent and Application Environment. GUI provides basic support to show the influences invoked by the agents and the messages sent by the agents.

Developing an application with a software environment starts with the implementation of the various hot spots of the Agent and Application Environment package (we discuss the hot spots below). SystemCreator then integrates the hot spots with the framework core to build the application. SystemCreator creates the application environment and populates it with the agents. SystemCreator returns an instance of SystemManager that is used to control the execution of the application. SystemManager allows the user to start the application, to suspend and resume the execution, and to terminate the application.

To develop an application with agents deployed in a physical environment, only the hot spots of the Agent package have to be implemented and integrated with the framework core (Agent package). The integrated software can directly be deployed on the physical machines. To enable the agents to interact with the physical environment, the software has to be connected to sensors and actuators.

\subsection{Overview of the Agent Package}

Figure 3 shows a general overview of the Agent package. The package is divided in several sub-packages, we briefly explain each of the sub-packages in turn.

KnowledgeIntegration encapsulates the agent's internal state that is modelled as a collection of knowledge objects (KnowledgeObject). Besides basic support for adding and removing knowledge objects, KnowledgeIntegration provides 


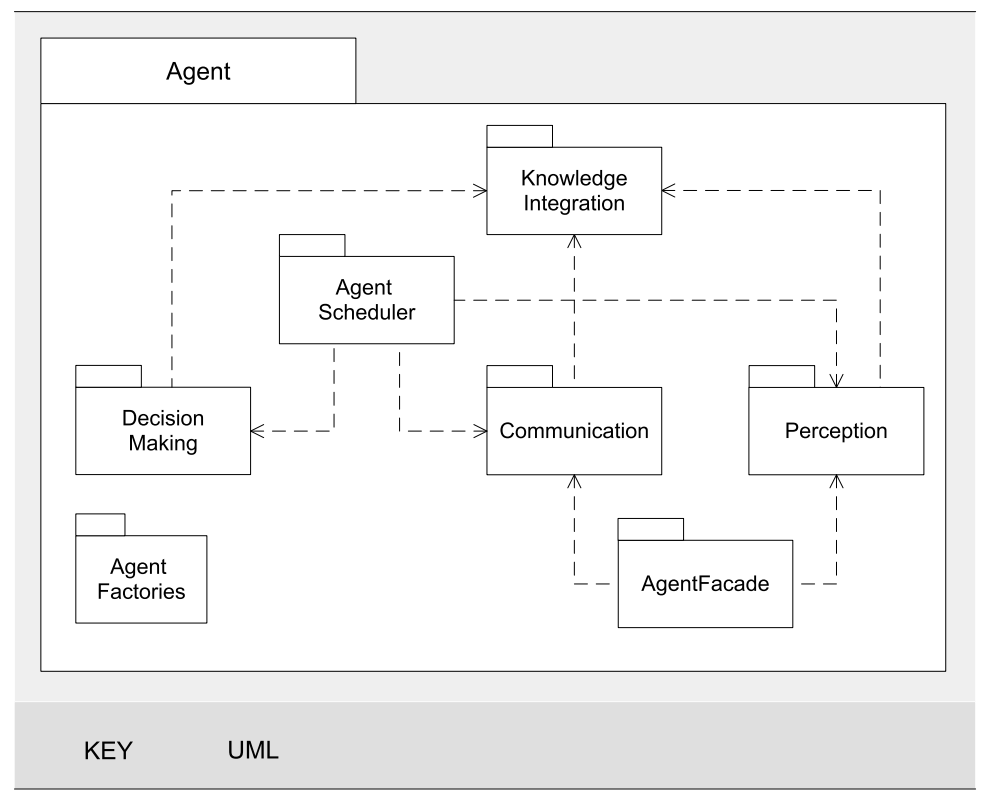

Fig. 3. General overview of the Agent package

various additional features such as support to update the state with a given set of knowledge objects, selection of the knowledge objects of a particular type, registration of an observer to notify changes of a selected type of knowledge objects, etc.

Perception enables the agent to sense the environment. Perception supports selective perception, i.e. agents can sense the environment with a set of selected foci (Focus), interpret representations with descriptions (Description), and filter the resulting Percept with a set of selected filters (Filter) [33]. As an example, Visualfocus that extends Focus enables agents in the Packet-World to visually perceive their environment with a given perception range.

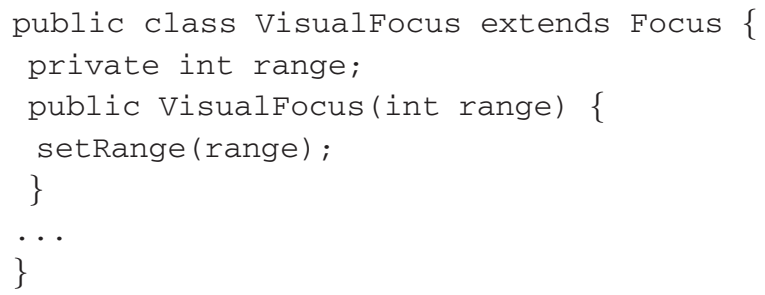

Agents can select foci and filters during decision making and communication. The selected foci and filters are registered in KnowledgeIntegration and used by Perception to sense the environment. Perception interacts with the environment via a set of sensors (Sensor). For agents situated in a software environment, a sensor is an abstraction that provides an interface with the application environment. Software 
agents receive the representation of a perception request via the AgentFacade. For agents situated in a physical environment, a sensor is the physical device the agent uses to sense the surrounding world.

Communication deals with the communicative interactions of the agent. Agents communicate according to well-defined communication protocols (Protocol) [32]. A protocol consists of a series of protocol steps. We distinguish between three types of protocol steps: conversation initiation, conversation continuation, and conversation termination. A conversation initiation step starts a new conversation according to a particular protocol. An agent initiates a conversation based on its current knowledge, possibly taking into account the data of a message received from another agent that started the interaction. A conversation continuation performs a step in an ongoing conversation. A conversation continuation may deal with a received message without directly responding to it, it may immediately react with a reply message, or it may pick up a conversation after a break. Finally, a conversation termination concludes an ongoing conversation. The termination of a conversation can be induced by changing circumstances in the environment or it can directly result from a preceding step of the conversation. In addition to these protocol steps, the framework provides support for time-outs. The developer can associate a time duration to a conversation, together with a reaction. When no activity has occurred in the conversation for the specified time duration, the accompanying reaction will be executed. For example, if an agent does not receive an answer to a request within a particular time window it can repeat the request, it can discard the conversation, or it can react in some other way. Messages are exchanged in ExternalMessage format. An ExternalMessage encapsulates a message as a plain string. Internally agents use AgentMessage to represent a message. Instances of AgentMessage encode messages in terms of domain objects. The conversion of messages is handled by the SLDecoderEncoder and is based on a domain Ontology. "SL" stands for Semantic Language and is defined by FIPA [16]. For the implementation of the SLDecoderEncoder, we reused a package of the Jade libraries [7]. Ontology represents the common vocabulary agents use to communicate with one another. Each concept that agents want to use as content of a message needs to be included in their ontology. A concept is stored in the vocabulary as a tuple of the class of the concept and an external name used to refer to the concept (Tuple<String, Class >). For example, for agents in the Packet-World, the ontology is defined as:

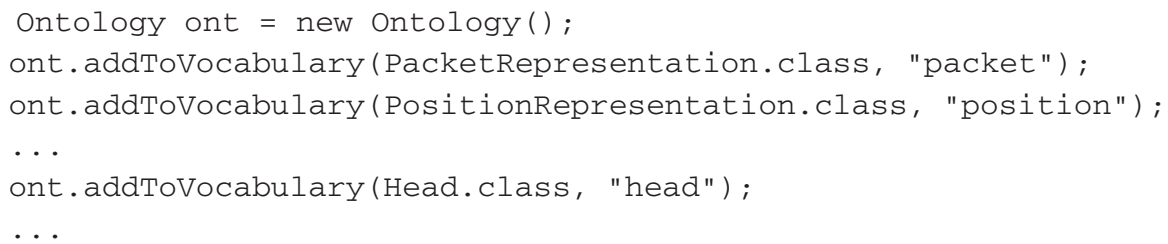

Communication is equipped with a Transceiver to exchange messages with other agents. For agents situated in a software environment, the transceiver is an abstraction that provides an interface with the application environment. Software agents receive incoming messages via the AgentFacade. For agents situated in a physical 
environment, the transceiver is the physical device the agent uses to communicate with other agents in their neighborhood.

Decisionmaking encapsulates a behavior-based action selection mechanism that supports roles (Role) and situated commitments (SituatedCommi tment) [3122]. The framework offers the application developer the predefined FreeF I ow package to instantiate free-flow trees. We elaborate on decision making with a free-flow tree in Sect.4 Decision making results in the selection of an Operator that is passed to Execution. An operator is an internal representation used by the agent to represent a selected action. Execution decouples the agent's internal representation of actions from the influences that are available to the agent to access and modify the environment. For agents situated in a software environment, the execution module is an abstraction that converts the operator into an Influence that is invoked in the application environment. For agents situated in a physical environment, the execution module interfaces with the physical device the agent uses to act in the environment, such as a switch or a motor.

AgentScheduler encapsulates the thread of the agent. AgentScheduler determines when the different modules (perception, decision making, and communication) get control. Decision making and communication can select foci and filters that are used by perception to perceive the environment when it gets control. Scheduling of the various activities can be customized according to the requirements at hand. The framework offers a default schema LTDSchedule (Look-Talk-Do [25]) that extends AgentSchedule. LTDSchedule successively activates perception, communication, decision making in an endless loop.

AgentFactories is a package that supports the creation of agents. AgentFactories consists of two sub-packages: SoftwareAgentFactory and PhysicalAgentFactory that can be used to create agents situated in a software environment and a physical environment respectively. In particular, the PhysicalAgentFactory supports the instantiation of robot software with the Lego-Mindstorms package [3].

Hot Spots. The hot spots of Agent can be divided in two groups: hot spots related to the interaction of the agent with the environment, and hot spots related to the agent's behavior.

Hot spots related to the interaction with the environment are only applicable for agents situated in a physical environment and include Sensor, Transceiver, and Execution. For a concrete application, these hot spots have to be instantiated to interface with the appropriate physical devices. For agents that live in a software environment the core of the framework encapsulates general implementations for sensor, transceiver and execution that are used for the interfacing with the application environment. We illustrate hot spots related to the interaction with the environment for a robot in Sect. 7

Hot spots related to the behavior of the agent determine how an agent perceives the environment, how it selects actions, and how it communicates with other agents. The hot 
spots include: Knowledgeobject, Focus, Description, Percept, Filter, DecisionMaking, Role, SituatedCommitment, Operator, Ontology, Protocol, and AgentSchedule. We illustrate a number of instances of these hot spots in Sect. 4

\subsection{Overview of the Application Environment Package}

Figure 4 shows a general overview of the application environment package. We briefly look at the various sub-packages.

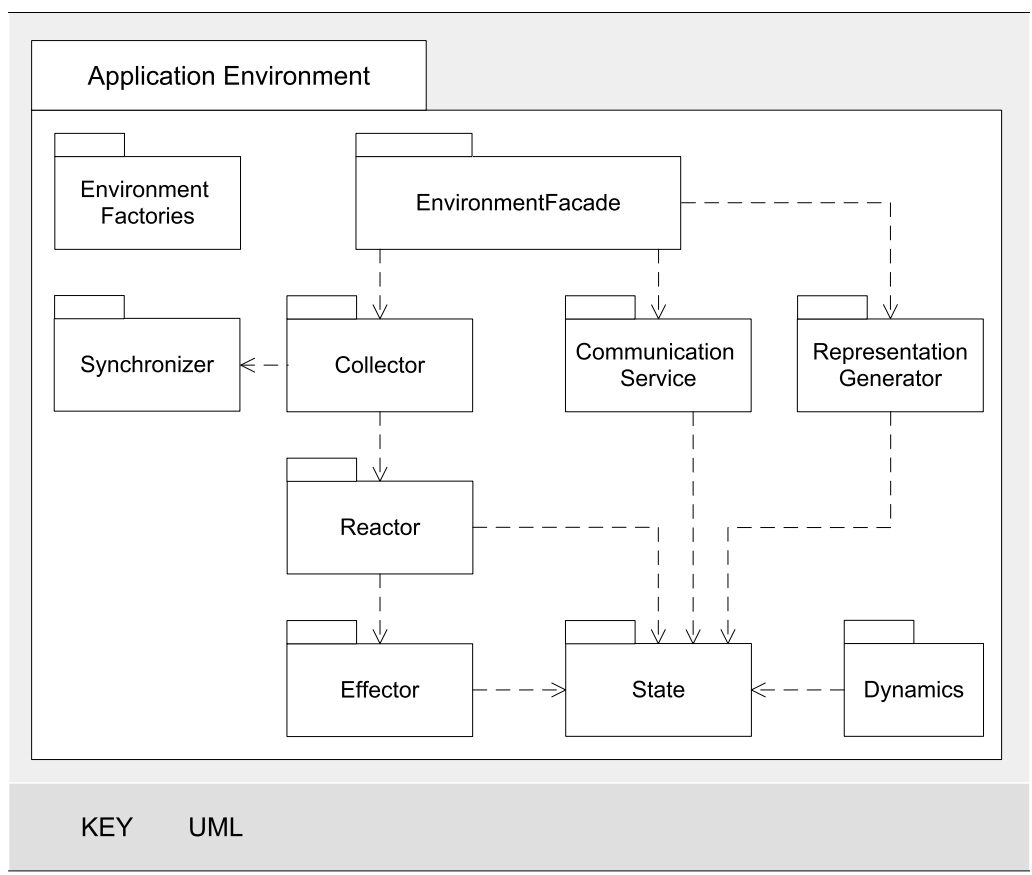

Fig. 4. General overview of the Application Environment package

EnvironmentFacade shields the internals of the application environment to agents. The facade provides an interface to agents to sense the application environment, to invoke influences, and to send messages. EnvironmentFacade dispatches the various activities for processing to the appropriate modules.

State encapsulates the actual state of the application environment. The state of the application environment includes a representation of the topology of the environment, state of static and dynamic objects, external state of agents (e.g., identities and positions), and state of environmental properties that represent system-wide characteristics. An example of an environmental property in the Packet-World is a gradient field that guides agents to a battery charger. State in the framework is set up as a collection of Item objects and a collection of Relation objects. Item is an abstraction 
for elements in the application environment, with StaticItem and DynamicItem as specializations. The state of a StaticItem is invariable over time, state of a DynamicItem may change over time. Relation represents a relation between two or more Item objects. An example of a relation in the Packet-World is an agent that holds a packet. The framework supports various methods to observe and manipulate Item and Relation objects.

Dynamics encapsulates a collection of ongoing activities (OngoingActity). An ongoing activity defines the behavior of a DynamicItem taking into account the current state of the application environment. Ongoing activities can be created at system startup and exist for the full lifetime of the application, or they can be created and destroyed dynamically at runtime. An example of an ongoing activity in the PacketWorld is the maintenance process of digital pheromones. OngoingActity is supplied with an OngoingActivityschedule that encapsulates a thread to execute the ongoing activity. Developers can extend OngoingActivitySchedule to define application-specific behavior for ongoing activities.

Representationgenerator is an active module that generates representations (Representation) of the state of the application environment for agents. Representations are generated according to perception requests and the applicable perception laws that govern what the agents can observe. RepresentationGenerator applies the perception laws in a strict sequence. The definition of the ordering is a responsibility of the developer. A concrete perception law is defined as a subclass of PerceptionLaw and must implement the method:

public abstract Representation enforce (AgentId observer,
Representation representation, Vector<Focus> foci, State state);

A concrete perception law puts application specific restrictions on the representation generated for an observer, given the set of selected foci and the current state of the application environment. The following example illustrates a perceptual law for the Packet-World:

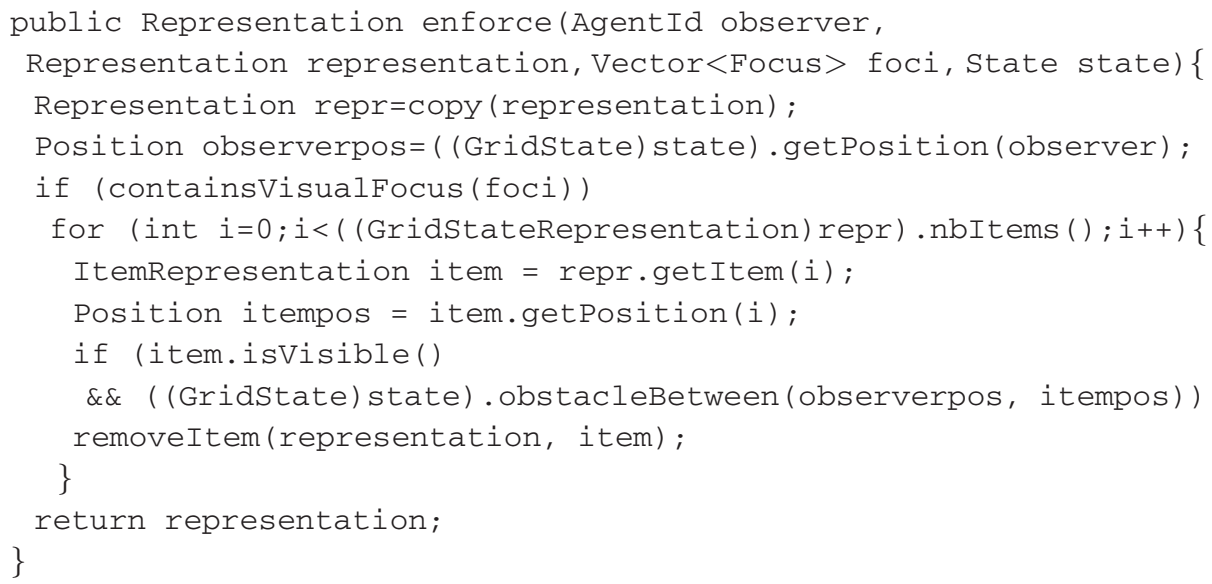


This law removes all the visible items in a representation that are out of the view of an observer due to an obstacle.

Communicationservice is an active module that handles message transport through the environment. Messages are delivered first-in-first-out. The application developer can define communication laws that enforce domain specific constraints on the transport of messages. A concrete communication law is defined as a subclass of CommunicationLaw and must implement the method:

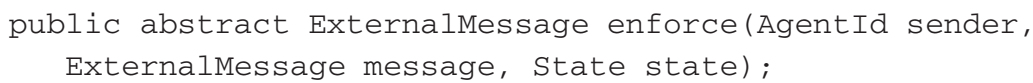

A typical example is a communication law that restricts the delivering of messages to a specific distance from the sender:

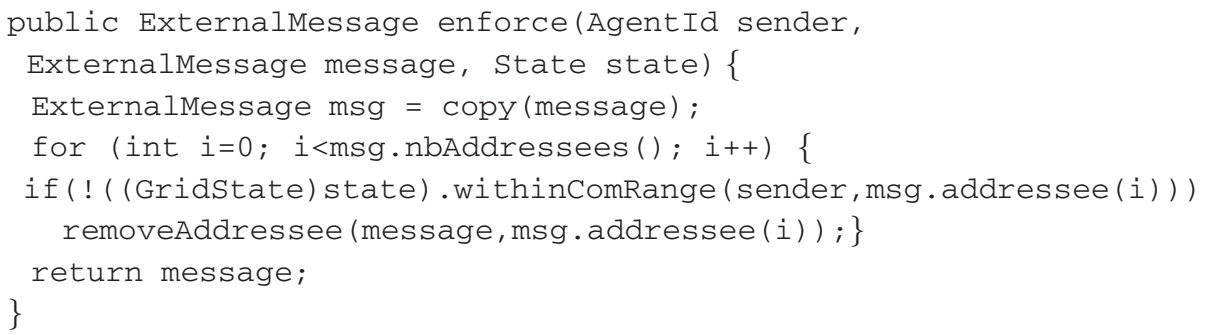

This laws drops all addressees for a given message that are not within communication range of the sender.

Synchronizer determines the type of synchronization of simultaneous actions. Simultaneous actions are actions that happen together and that can have a combined effect in the application environment. An example of simultaneous actions in the PacketWorld are two agents that push the same packet in different directions. As a result, the packet moves according to the resultant of the two actions. Ferber and Müller have introduced a model for simultaneous actions in which all the agents of the multiagent system act at one global pace, i.e. the agents are globally synchronized [15]. In this model, the environment combines the influences of all agents in each cycle to deduce a new state of the environment. We have introduced a model for regional synchronization [26 2927]. With regional synchronization agents form synchronized groups — regions — on the basis of their actual locality. Regions are disjoint sets of agents, i.e. at any time each agent is a member of exact one region. Different regions act asynchronously, while agents act synchronously within their region. Regional synchronization complies with the basic characteristic of locality of situated agents. We elaborate on support for simultaneous actions in Sect.5.

The framework includes support for three types of synchronization: (1) NoSynchronization, i.e. agents act asynchronously which implies that there is no support for simultaneous actions; (2) GlobalSynchronization, all agents act at a global pace-i.e. the Ferber-Müller model; and (3) Regional Synchronization, i.e. agents act simultaneously based on their actual locality. 
Collector is an active module that collects the influences (Influence) invoked by agents. Collector uses a Synchronizer to determine the sets of synchronized agents. If no synchronization is provided, the collector directly passes the influences to the reactor. With global synchronization, the collector collects the influences of all agents in the system before it passes the complete set to the reactor. With regional synchronization, the collector passes sets of influences per region to the reactor. Collector encapsulates its own thread, so that it can execute influences in parallel with other activities in the application environment.

Reactor and Effector. Reactor is responsible for processing sets of synchronized influences. The reactor calculates the effects of the influences according to current state of the application environment and the action laws of the multiagent system. This results in a set of effects (Effect). Effector is responsible for executing the effects of the influences resulting in state changes in the application environment. We elaborate on interaction in the application environment with the Collector-ReactorEffector chain in Sect.5.

EnvironmentFactory is a package that supports developers with the creation of an application environment for the multiagent system. EnvironmentFactory creates the internals of the application environment, it initializes the state of the environment with items and relations between items, it integrates the laws for perception, action, and communication, and it allows the developer to specify a synchronization approach for the application.

Hot Spots. Hot spots of the application environment include: State with StaticItem, DynamicItem and Relation, OngoingActivity, Representation, Influence, and Effect. Besides, PereceptionLaw, ActionLaw, and CommunicationLaw are hot spots that have to be defined for the application at hand. Finally, Synchronizer is a hot spot of the application environment for which the developer can simply select one of the available synchronizers.

\section{Decision Making with a Free-Flow Tree}

The framework provides the developer with one predefined behavior-based action selection mechanism: free-flow trees. Implementations of other decision making mechanisms are not provided in the current framework implementation and as such have to be defined by the developer. In this section, we explain how free-flow trees extended with roles and situated commitments are supported by the framework. But first, we introduce the notion of a free-flow tree.

\subsection{Free-Flow Trees}

A free-flow tree is a behavior-based action selection mechanism that was introduced by Rosenblatt and Payton in [21]. Tyrrell [23] has demonstrated that hierarchical free-flow architectures are superior to flat decision structures, especially in complex and dynamic 
environments. The results of Tyrrell's work are recognized in recent research, for a discussion see [9].

A free-flow tree is a hierarchy composed of activity nodes (in short nodes) which receive information from internal and external stimuli in the form of activity. The nodes feed their activity down through the hierarchy until the activity arrives at the action nodes (i.e. the leaf nodes of the tree) where a winner-takes-all process decides which action is selected. A free-flow tree allows an agent to take different preferences into consideration simultaneously. For example, consider an agent in the Packet-World that spots two candidate packets to be picked at about equal distance. A Packet-World agent also has to maintain its battery. To move to the battery charger, the agent can follow the gradient of the field emitted by the charger. If the agent is only able to take into account one preference at a time it will select one packet and move to it, or alternatively it will follow the gradient towards the battery charger. With a free-flow tree the agent can move towards one packet while it moves in the direction of the charge station, i.e. if the agent needs to recharge its battery in the near future, it will move towards the packet that is nearest to the battery charger.

Free-flow trees are developed from the viewpoint of individual agents. To enable agents to exhibit explicit social behavior, we have extended the free-flow architecture with the abstractions of a role and a situated commitment [31|22]. Fig. 5] shows a free-flow tree for an agent in the Packet-World extended with roles and situated commitments.

A role represents a coherent part of functionality of an agent in the context of an organization. Roles provide building blocks for social organization in a multiagent system. Agents are linked to other agents by the roles they play in the organization. A role can consist of a number of sub-roles, and sub-sub-roles of sub-roles etc. A role matches to a sub-tree in the free-flow tree. For the Packet-World agents, three main roles are distinguished: Individual, Chain, and Maintain. In the role Individual, the agent performs work, independent of the other agents. The agent searches for packets and brings them to the destination. The Chain role is composed of two sub-roles: Head and Tail denoting the two roles of agents in a collaboration to pass packets along a chain. Finally in the Maintain role, the agent recharges its battery.

A situated commitment defines a relationship between one role (the goal role) and a non-empty set of other roles (the source roles) of the agent. When a situated commitment is activated the behavior of the agent tends to prefer the goal role of the commitment over the source role(s). Favoring the goal role results in more consistent behavior of the agent towards the commitment. In a collaboration agents commit relatively to one another, typically via communication. However, an agent can also commit to itself, e.g. when it has to fulfill a vital task. A situated commitment is represented in the free-flow tree by a connector that connects the source roles of the situated commitment with the goal role. When a situated commitment is activated, extra activity is injected in the goal role relative to the activity levels of the source roles. The connector Charging in Fig. 5 denotes the situated commitment of an agent to itself to recharge its battery. Charging connects the top nodes of the source roles Individual and Chain with the goal role Maintain. The connectors HeadOfChain and TailOfChain denote the mutual situated commitments of two agents that collaborate to pass packets in a chain. 


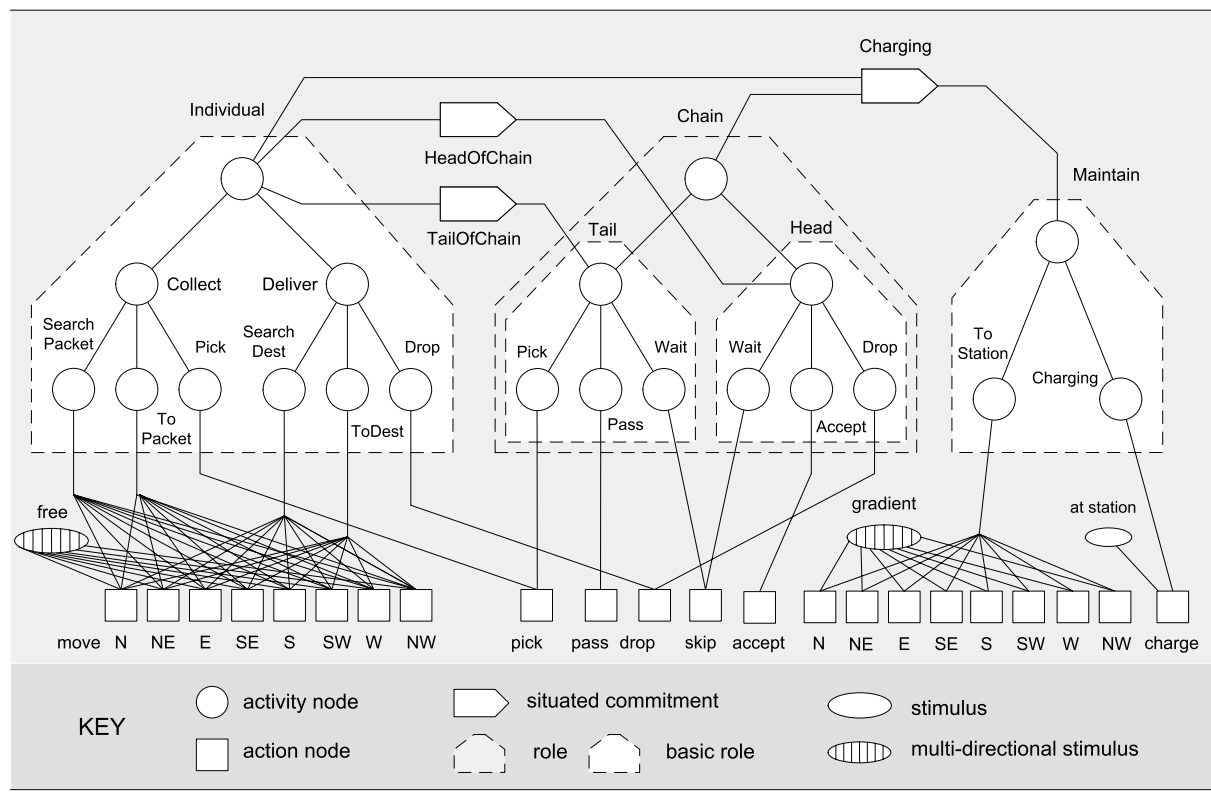

Fig. 5. Free-flow tree for a Packet-World agent with roles and situated commitments (details such as stimuli of activity nodes are omitted; action nodes with the same name-i.e. the move actions - need to be joined together; free and gradient are multi-directional stimuli that have a value for each of the eight directions the agent can move to)

\subsection{Support for Free-Flow Trees in the Framework}

Figure 6 shows the main classes of the FreeFlow package of the framework. A freeflow tree consists of three types of tree elements (TreeElement): Node, Stimulus, and SituatedCommitment. Tree elements are connected through links (Link). A tree element receives an amount of activity (represented by Activity) of its parent elements and can inject activity in its child elements. Each link has a weight factor that determines how much of the injected activity is passed along that link. Stimulus has no parent elements but calculates its activity based on the internal state of the agent. SituatedCommitment is connected with a non-empty set of nodes that represents its source roles, and one particular node that represents its goal role. A situated commitment can be triggered by two conditions: one that activates the commitment and one that deactivates it. In the activated state, the commitment combines the activity receive from its source roles with an AdditionFunction and injects the resulting activity in its goal role. Node is further specialized in ActivityNode and ActionNode. An ActivityNode is a regular node of the tree, an ActionNode is a leave node of the tree that is associated with an operator. Each Node has a CombinationFunction that determines how the activity received from its parent nodes is combined. Activity nodes that represent top nodes of a role have to implement the Role interface that associates an explicit name with the role. A FreeFlowTree represents the system node 


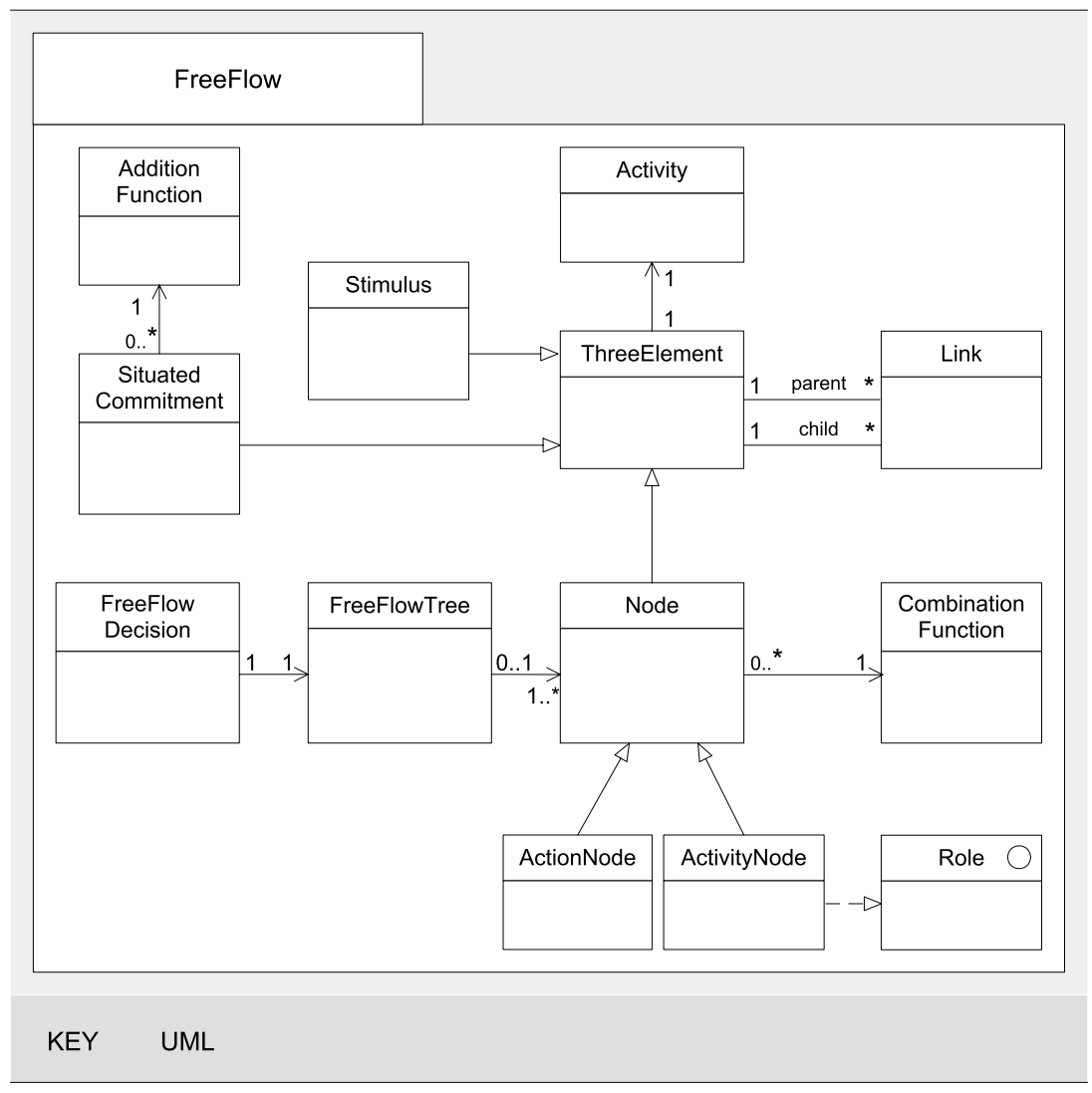

Fig. 6. Overview of the Free-flow package

and is the entry point for FreeFlowDecision that implements the action selection algorithm.

Hot Spots. To build a concrete free-flow tree a number of hot spots have to be implemented. In particular, Activity, stimulus, situatedCommitment, Additionfunction and CombinationFunction, ActionNode, Role, and Link are hot spots. The framework supports the developer with various basic implementations for most of these hot spots. BasicActivity is a subclass of Activity that represents a basic representation of activity by means of a double value. More advanced implementations have to be defined by the developer. The framework supports the definition of simple stimuli (SimpleStimulus) as well as multi-directional stimuli (Vectorstimuli). A situated commitment has to be defined as a subclass of SituatedCommitment and requires the definition of an activation condition, a deactivation condition, and the definition of the outcome of the situated commitment when 
it is activated. This latter requires the definition of a concrete AdditionFunction. As an example, we illustrate the definition of the situated commitment Charging of a Packet-World agent:

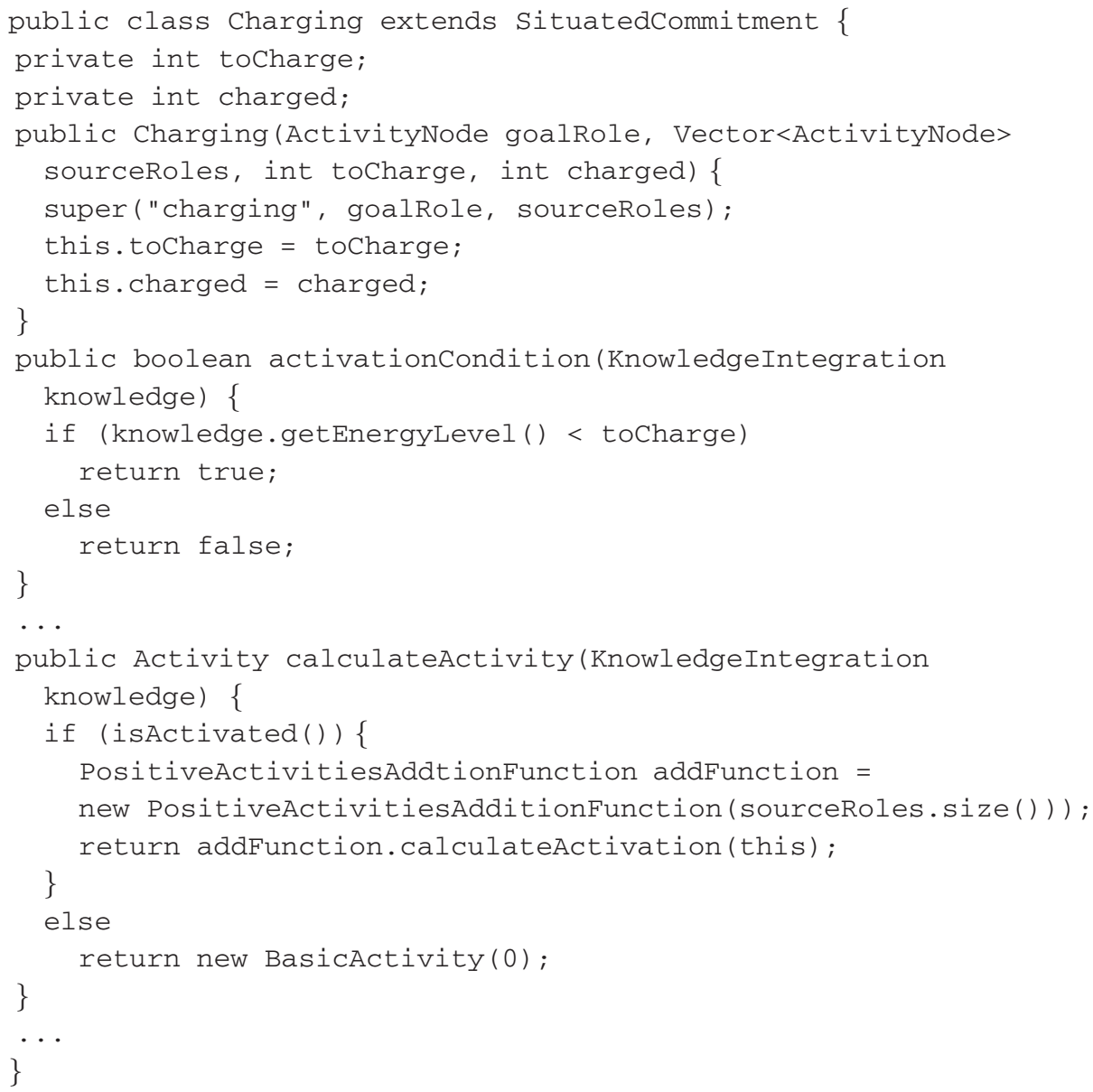

For each Node a CombinationFunction has to be defined. The framework supports various basic functions, including AddFunction, MultiplyFunction, and MaximumFunction. ActionNode is a hot spot, for each action node of the tree a subclass of ActionNode has to be defined. Such subclass must implement the method getoperator ( ). This method returns the operator that is executed by the agent if that particular node is selected for execution. Each Link in the tree has to be assigned a weight factor, except if the weight factor has the default value of 1 . Finally, a subclass of FreeFlowDecision must be defined. This subclass must implement the abstract method createFreeFlowTree ( ) that constructs the application specific free-flow tree.

The framework's cookbook [34] provides examples of the various hot spots to support the developer with the instantiation of a free-flow tree. [22] describes a supporting 
modeling language and design process to design free-flow trees with roles and situated commitments.

\section{Simultaneous Actions in the Environment}

An interesting feature provided by the framework is support for simultaneous actions. Support for simultaneous actions enables to simulate the effects of actions that are conceptually executed at the same time, but physically are performed separated in time, e.g., on a single or sequential processor system. In this section, we first explain the notion of simultaneous actions. Then, we show how simultaneous actions are supported in the framework. We illustrate the explanation with examples from the Packet-World.

\subsection{Simultaneous Actions}

In the literature, several researchers refer to simultaneously performed actions. Some examples: Allen and Ferguson [5] discuss "actions that interfere with each other" and that can have "additional synergistic effects". Boutilier and Brafman [8] mention "concurrent actions with a positive or negative interacting effect". Griffiths, Luck and d'Iverno [17] introduce the notions of "joint action that a group of agents perform together" and "concurrent actions, i.e. a set of actions performed at the same time". Joint actions and concurrent actions are based on the concepts of "strong and weak parallelism" introduced Kinny [19]. In [20], Michel, Gouaïch, and Ferber introduce the notions of weak and strong interactions that are related to simultaneous actions.

We denote simultaneous actions as actions that happen together and that can have a combined result. To calculate the effects of simultaneously performed actions that physically are performed separated in time, the actions are reified as influences [12]. Support for simultaneous actions requires two mechanisms: first, a mechanism is needed that determines which influences are treated as being executed together; second, a mechanism is needed that ensures that the combined outcome of simultaneously performed influences is in accordance with the domain that is modelled.

Determining Simultaneity. Simultaneity of influences is determined by a synchronization mechanism. Two possible mechanisms for synchronization are global synchronization and regional synchronization. With global synchronization, all agents in the multiagent system act simultaneously. Global synchronization is simple to implement, but the mechanism imposes centralized control. Regional synchronization offers more finegrained synchronization. With regional synchronization, the composition of groups of synchronized agents_-regions — depends on the actual locality of the agents and dynamically changes when agents enter or leave each others locality. Support for regional synchronization can be implemented as a service of the application environment [2610]. Alternatively, the agents can take care for the formation of regions themselves, providing a fully decentralized solution for synchronization. [29]27] discuss an algorithm for regional synchronization in detail and provides a proof of correctness. 


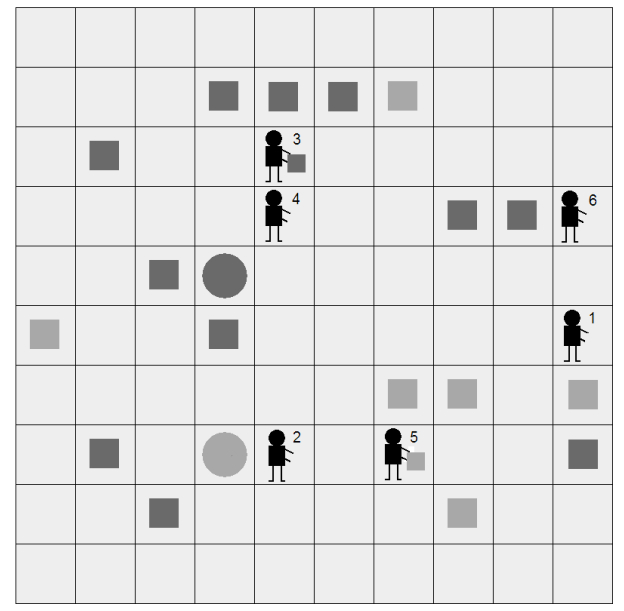

Fig. 7. Example of simultaneous actions in the Packet-World

Imposing Domain Constraints. Domain constraints are imposed through a set of action laws. Action laws determine the effects of a set of synchronized influences on the state of the application environment. As such, action laws impose constrains on the implications of agents' (inter)actions. Figure 7 shows an example of simultaneous actions in the Packet-World. In the depicted situation, agents 3 can pass packets to agent 4 that can directly deliver the packets at the destination. Such packet transfer only succeeds when the two agents act together, i.e. agent 3 has to pass the packet while agent 4 simultaneously accepts the packet. To model the packet transfer, an action law is defined. This definition includes:

1. The set of influences. This set consists of two influences: PassInfluence and AcceptInfluence.

2. The preconditions. The packet transfer only succeeds if: (i) both agents have enough energy to execute the transfer, (ii) the locations of the agents match with a chain, (iii) the tail holds a packet and the head does not.

3. The effects. Applying the law properly reduces the energy level of both agents, and the packet is transferred from tail to head.

Notice that agent 2 and 5 also form a chain to transfer packets. In this chain however, packets are passed indirectly via the environment, i.e., agent 5 can put packets in between the two agents and agent 2 can pick the packets and deliver them at the destination. Contrary to the synchronous collaboration between agent 3 and 4, this asynchronous collaboration does not involve any simultaneous actions.

\subsection{Support for Simultaneous Actions in the Framework}

Figure 8 shows the main classes of the framework involved in the execution of simultaneous actions. 


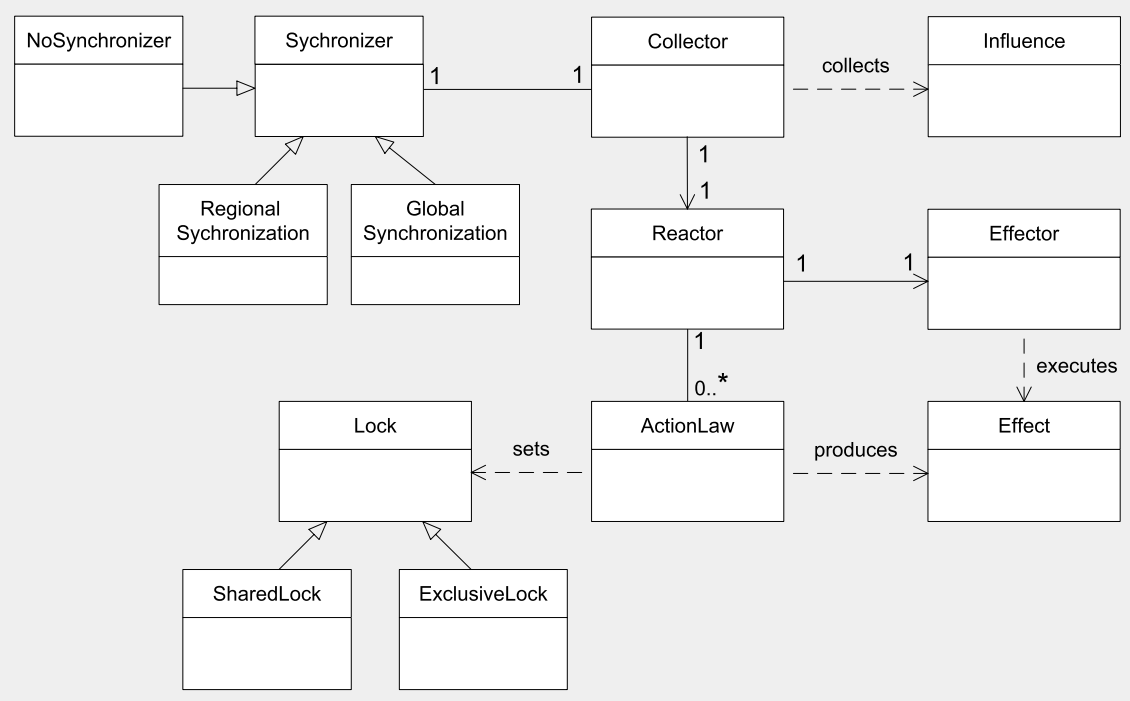

KEY UML

Fig. 8. Main classes of the framework involved in the execution of simultaneous actions

Collector collects the influences (Influence) invoked by the agents and stores the influences in a buffer. Domain specific influences are defined as subclasses of Influence. A simple example is StepInfluence that is defined as follows:

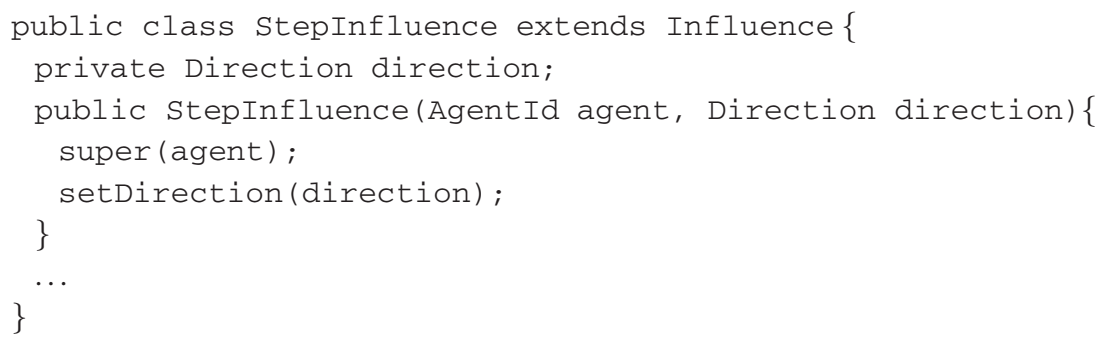

Synchronizer determines when influences are passed to the Reactor for execution. With Nosychronizer influences are passed one by one; with Globalsynchronizer the set of influences of all agents is passed; with RegionalSynchronization the influences are passed to the reactor per region. To form regions, the framework provides a default implementation for locality that is based on the default range of perception. In particular, a region in the framework consists of the set of agents that are located within each other's perceptual range, or within the perceptual range of those agents, and so on. Applied to the situation in Fig. 7 with NOSynchronizer all agents act asynchronously (in this case there is no 
support for passing packets directly from one agent to another); with Globalsynchronization all agents act at one global pace; with RegionalSynchronization each agent act simultaneously with the other agents within its region. If we assume a perceptual range of two fields, than there are three regions: agents 3 and 4, agents 2 and 5, and agent 1 and 6 . If in the depicted situation agent 1 makes a step towards South-West it enters the region of agents 5 and 2, while the original region of agent 1 and 6 is than reduced to only agent 6 .

Reactor receives sets of influences from the Collector and calculates the effects (Effect), i.e., state changes in the application environment. Therefore, the reactor uses the sets of action laws (ActionLaw). The ordering in which laws are applied depends on the number of influences involved in the law. The law with the highest number of influences is applied first, then the law with the second highest number is applied, and so on. For laws with an equal number of influences, laws are applied in random order. Domain specific action laws have to be defined as subclasses of ActionLaw. This definition requires the implementation of four methods:

public $<$ T extends Influence>Vector $<$ Class $<$ T $>>$ getInfluenceTypes (); public boolean checkConditions (Vector<Influence> influences); public Vector<Effect> geteffects (Vector<Influence> influences); public Vector $<$ Lock> getLocks();

The method getInfluenceTypes ( ) returns a vector of influence types, one for each influence involved in the law. This method allows the reactor to check whether the law is applicable or not. For TransferPacketLaw that models the rules for agents to transfer a packet, getInfluenceTypes ( ) is defined as follows:

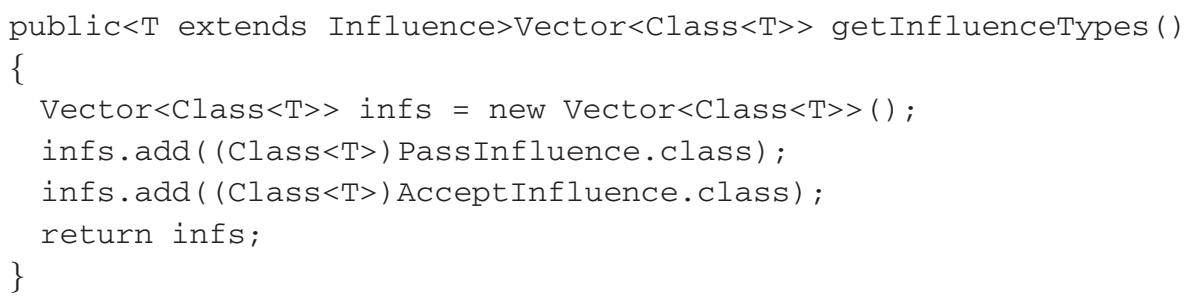

The method checkConditions ( ) verifies whether the necessary conditions hold to apply the law. For TransferPacketLaw the conditions are:

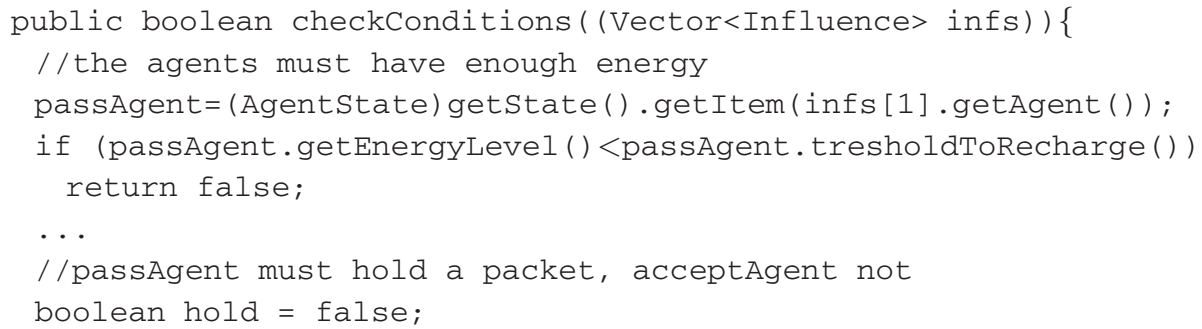




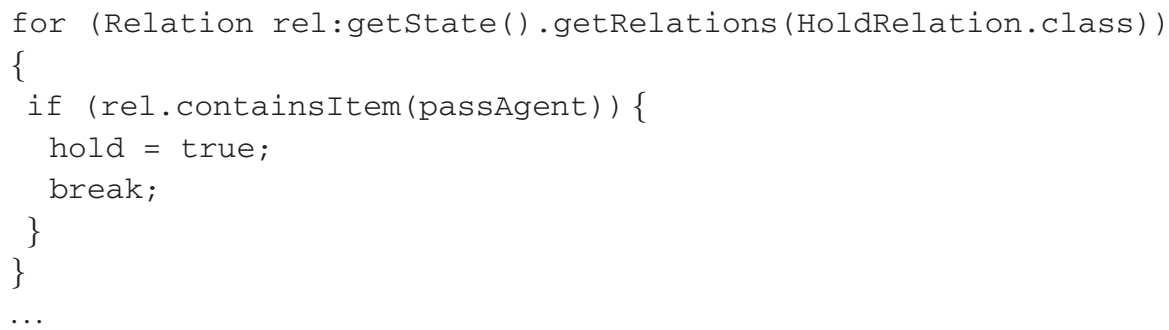

The method geteffects () returns the effects induced by the law. An application specific effect has to be defined as a subclass of Effect. A simple example is AddRelationEffect that is used to add a relation in the state of the application environment. Such relation is used to link the agent with the packet it accepts during a packet transfer:

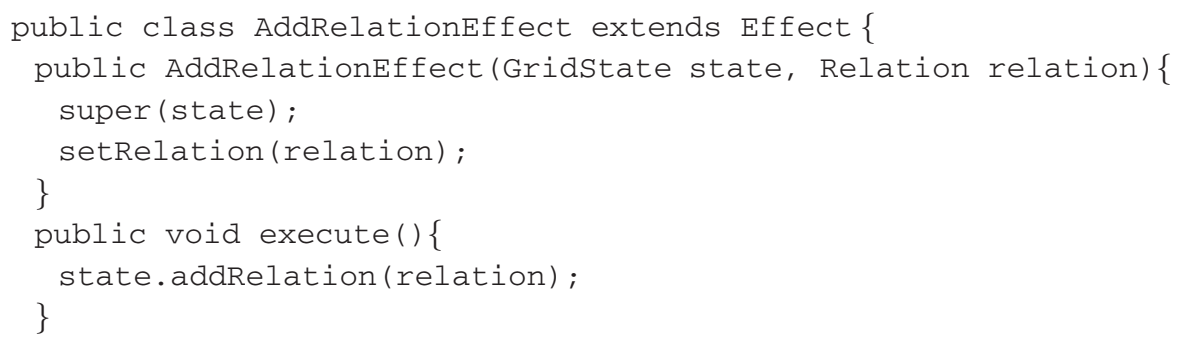

Finally, each action law has to implement the method getLocks ( ). This method returns the locks on the state elements used by the law. Locks (Lock) avoid conflicts between action laws. To ensure that all simultaneously performed influences are applied in the same circumstances, the action laws produce the effects of influences from the same state of the application environment. However, applying a law may induce constraints on state elements. For example, assume that StepLaw handles the movement of a single agent. If agent 6 in Fig. 7 makes a step to South than agent 1 can no longer step to North. To avoid a conflict between the application of the law for both agents, the first application of StepLaw puts a lock on the field the agent moves to. During the execution of the law for the other influence of the region, the reactor uses the lock to check whether the StepLaw is applicable or not. The framework supports two types of basic locks: ExclusiveLock and SharedLock. An ExclusiveLock on a state element of the application environment excludes other laws to access the locked element. A SharedLock allows other laws to put a shared lock on the element, however, it excludes a possible ExclusiveLock.

Effector is responsible to apply the effects induced by the action laws. Each Effect implements the method execute () that actually performs the effects to the state of the application environment, see the AddRelationEffect above. 
Hot Spots. Much of the complexity to deal with simultaneous actions is hidden by the framework core. If the application requires support for simultaneous actions, the developer has to select a particular type of synchronization. This selection has to be specified in the EnvironmentFactory definition. Furthermore, the developer has to define application specific instances for Influence, ActionLaw, Lock, and Effect, as illustrated above.

\section{Failure Treatment in the Framework}

Basic failure treatment in the framework is dealt with by Java exception handling. To avoid overloaded code excerpts, we have omitted the exception code in the examples in this paper. As an illustration, we show the exception handling for setting a lock in the SharedLockType class.

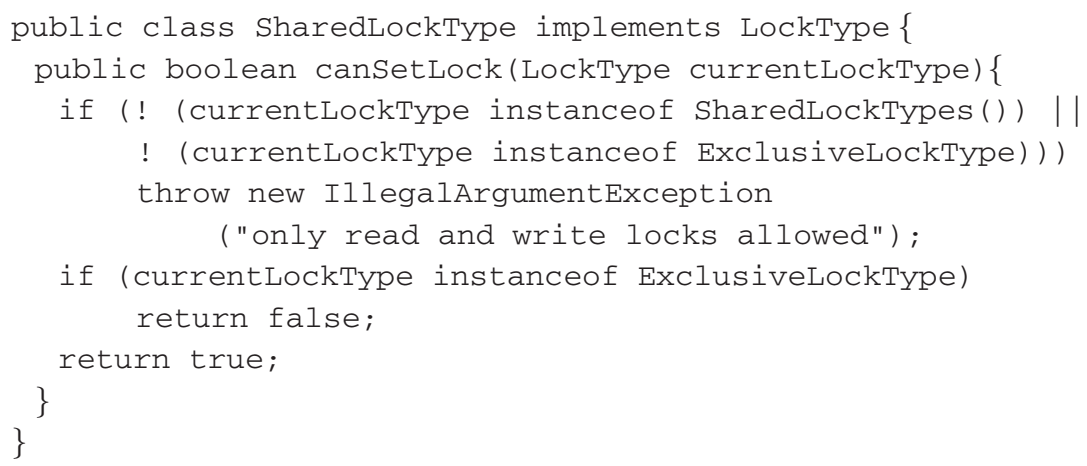

A shared lock can only be combined with another shared lock. The method public boolean canSetLock (LockType currentLockType) returns a boolean that indicates whether the type of lock can be set on a given object or not. When the given lock type is not a shared or exclusive lock, an exception is thrown. When there is already an exclusive lock on the object, the lock can no longer be set and a false is returned. Otherwise the lock can be set and true is returned.

\section{Applying the Framework to an Experimental Robot Application}

As a validation, we have used the framework to develop an experimental application in which two Lego-Mindstorms robots collaborate to organize the supply of products. In this section we give an overview of application. First, we introduce the application based on a simulation, and we explain the setup of the physical system with robots and the environment. Then we show how we have instantiated the framework to develop the application software. We limit the discussion to the agent software.

\subsection{Robot Application}

Figure 9 shows a schematical overview of the robot application. 


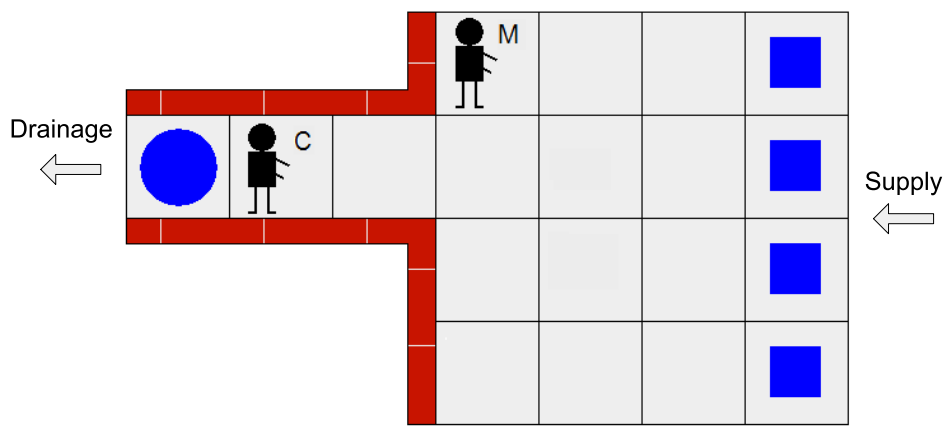

Fig. 9. Simulation of the robot application

The environment consists of two zones: the corridor on the left side in which a nonmobile crane robot can manoeuvre, and the rectangular factory flour on the right side in which a mobile robot can move around. The colored packets on the right side of the factory floor represent products. The circle represents the delivering point for products. The task of the robots is to guarantee a stream of products from supply to drainage.

We have developed the robots with the Lego-Mindstorms packet [3]. Besides building blocks to construct robots, Lego-Mindstorms offers a programmable microcomputer called Robotic Command eXplorer (RCX) to program a robot. To enable the robot to interact with the environment, various sensors (light, pressure, etc.) and actuators (switches, motors, etc.) are available that can be connected to the RCX. Furthermore, the RCX is equipped with an infrared serial communication interface that enables a developer to program the microcomputer. We have used the LeJOS (Lego Java Operating System), as a replacement firmware for the Lego Mindstorms RCX. LeJOS is a reduced Java Virtual Machine that fits within the $32 \mathrm{~kb}$ on the RCX, and that allows to program a Lego robot with Java [4].

Figure 10 shows one of the robots. Robots are equipped with various sensors to monitor the environment, and they have two grasp arms to pick up packets. The robots can communicate with a local computer via infrared communication.

Figure 11 shows environment with the two robots in action. The robots use light sensors to follow the paths that are marked by black lines.

\subsection{Applying the Framework}

Due to memory limitations of the RCX, it was not possible to execute the full robot control software directly on the robot hardware. Therefore the robot software is divided in two collaborating programs: one program running on the RCX of the robot that monitors the environment and executes actions, and a second program running on a local computer that selects actions. Figure 12 shows how the robot software is deployed on the various hardware units. The agents use LTDSchedule as scheduling schema. LTDSchedule is a predefined scheduling schema in the framework that successively activates perception, communication, decision making in an endless loop. Perception transforms the data sensed by InfraredSensor into a percept 


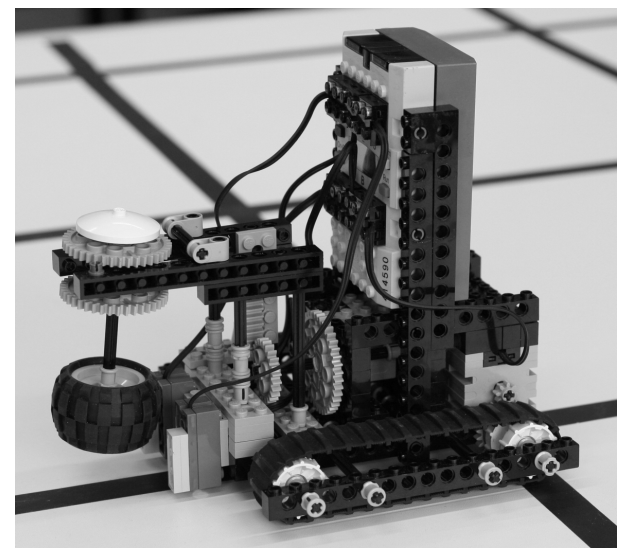

Fig. 10. A robot carrying a packet

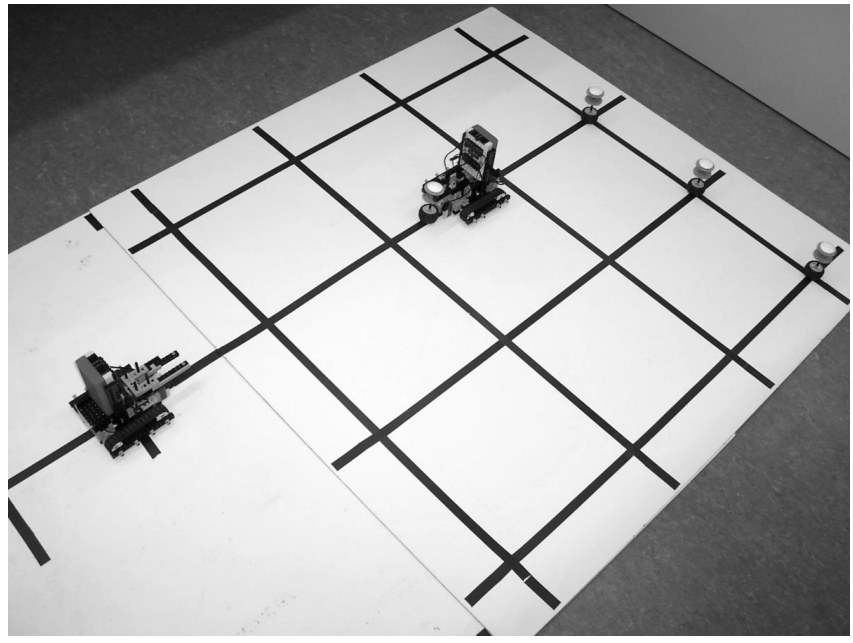

Fig. 11. The environment with the robots in action

(WAStatePercept). Periodically, the RCX sends an infrared message with the current status of the robot (position, hold packet or not) to the agent program on the computer. Infrared communication is handled by IRTower and IRPort on the host computer and the RCX respectively. The decision making modules (CraneAgentDecisionMaking and MobileAgentDecisionMaking) take care for action selection. The action selection mechanisms of the MobileAgent continuously executes a sequence of three roles: LookForPacket, ReturnToCorridor, and PassPacket. When the MobileAgent arrives with a new packet at the corridor, the CraneAgent executes AcceptPacket, it delivers the packet (DeliverPacket) at the destination, and subsequently waits for the next packet (Wait). WAExecution sends the selected actions to the RCX via the 


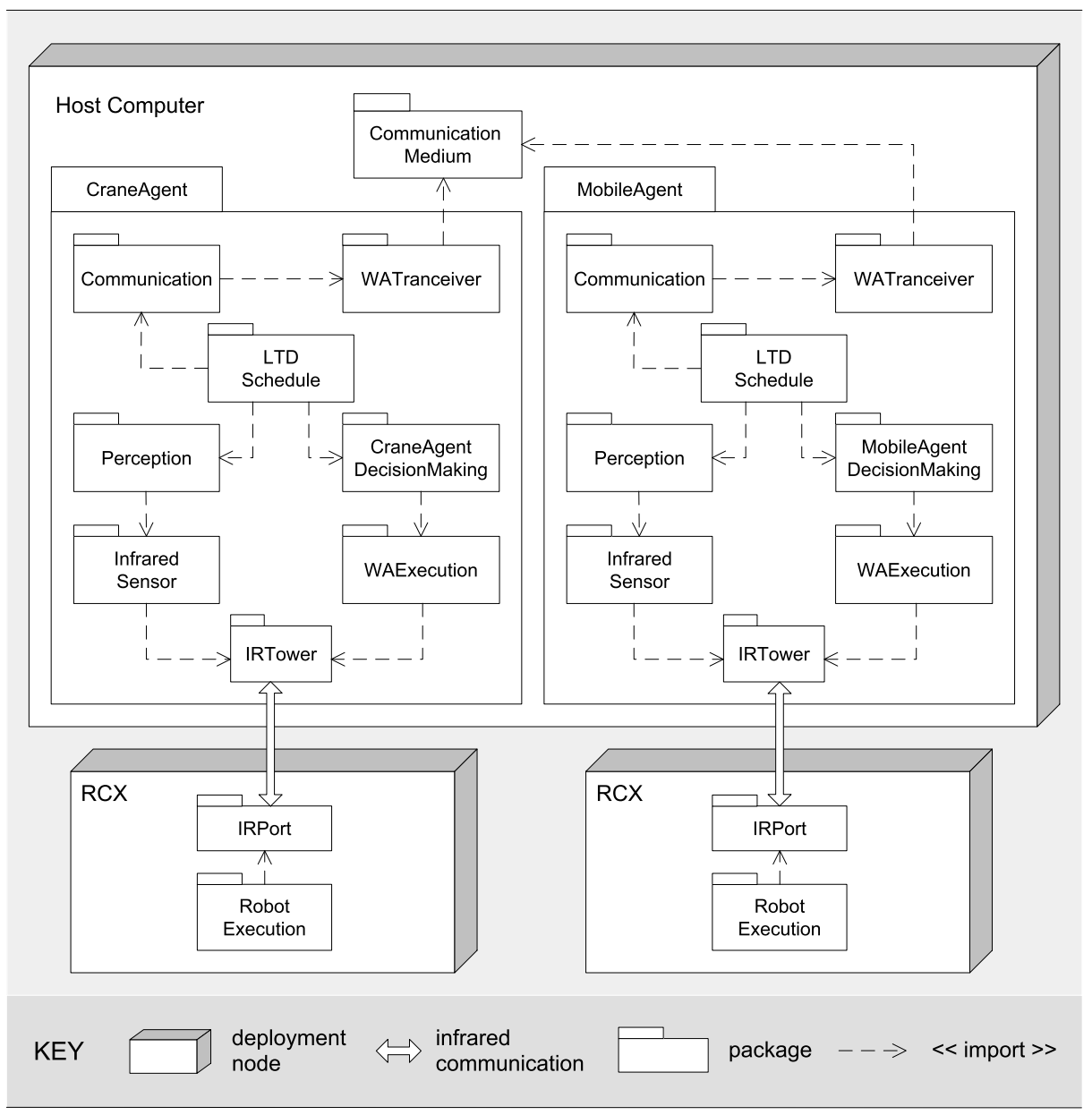

Fig. 12. Deployment of the robot software

IRTOwer. The decision making modules however, produce high-level actions, such as "drive to the corridor" and "put packet on the destination". When RobotExecution receives such an action, it translates the actions into low-level actions to steer the actuators.

When the MobileAgent arrives with a packet at the corridor, it has to pass the packet to the CraneAgent. To coordinate this interaction, the agents use a PassPacketProtocol that is handled by the Communication module. The subsequent steps of this protocol are depicted in Fig. 13. When the MobileAgent arrives at the corridor it informs the CraneAgent that it has arrived with a packet for delivering. The CraneAgent drives towards the packet and as soon as it is in the correct position, it informs the MobileAgent to release the packet. When the MobileAgent has released the packet it informs the CraneAgent. This latter then brings the packet to the delivering location. To communicate with one another, robots 


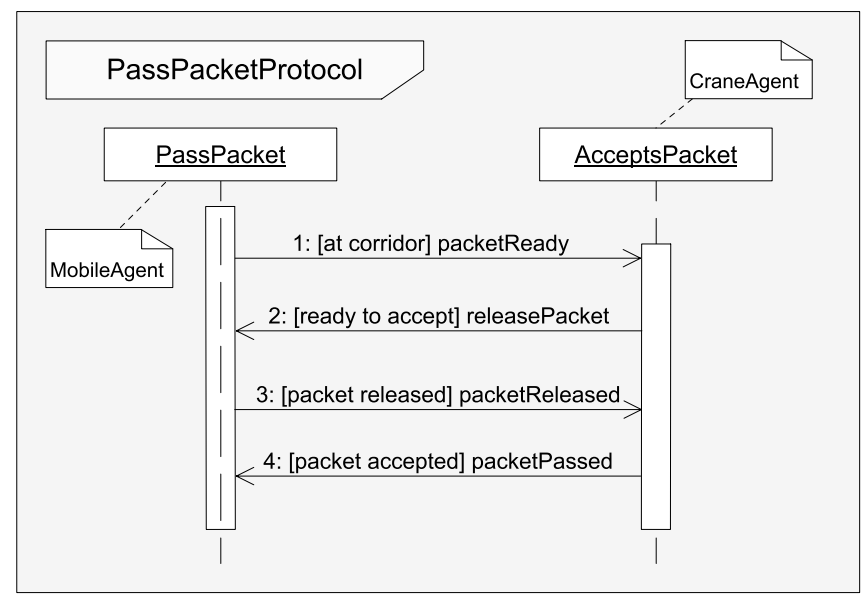

Fig. 13. Protocol to coordinate the transfer of a packet

use WATransceiver that transmits the messages via a simple CommunicationMedium.

Simulation of the Robot Application. To validate the application of the framework for a multiagent systems with a software environment, we also developed a simulation of the robot application (see Fig.96). In the simulated environment, the agents step on a grid. Products are represented by packets, and the drainage by a delivering point, similar as in the Packet-World. For the simulation, we were able to fully reuse the implementations of the agents, including the hot spots for perception, decision making, and the communication protocol for coordinating the packet transfer. The software agents also use the same scheduling schema as the robots. InfraRedSensor, WAExecution, and WATransceiver were no longer necessary for the agents in the software environment. The functionality for interfacing with the software environment is managed by the framework core.

\section{Differences with Other Multiagent System Frameworks}

Many frameworks and development tools for multiagent systems have been developed, for an overview see for example [2]. We touch on two representative examples and point to the typical differences with the framework presented in this paper.

JADE (Java Agent DEvelopment Framework [7]) is a well-known Java framework for the implementation of multiagent systems that fully complies to the FIPA specifications [16]. JADE comes with a set of graphical tools that supports the debugging and deployment of agent systems. JADE provides advanced support for agent communication in terms of ACL libraries and a distributed communication infrastructure. Support for developing agent internals is rather limited and environment functionality other that message communication infrastructure is absent. 
MadKit (Multi-Agent Development Kit [18]) is a modular multiagent system platform written in Java. Agents in MadKit can be programmed in Java, Scheme, Jess (rule based engine), or BeanShell. MadKit comes with a set of features for launching, displaying, developing and monitoring agents and organizations. MadKit provides extensive support for the AGR (Agent/Group/Role) organizational model: agents are situated in groups and play roles [13 14]. MadKit uses the notion of "system agent" to provide support for various kinds of system responsibilities that are typically covered by the application environment in the framework presented in this paper. As most multiagent system frameworks, MadKit is strongly biased towards a message passing interaction model. As such, support for other concerns such as perception and indirect interaction through the environment is limited.

\section{Concluding Remarks}

In this paper, we gave an overview of an object-oriented framework for situated multiagent systems. The framework targets experimental applications that are characterized by highly dynamic operation conditions and in which global control is difficult to achieve. The framework shows a concrete design of various mechanisms for adaptivity we have developed in our research, including selective perception, protocol-based communication, and behavior-based decision making with roles and situated commitments, and shows its application to an experimental robot application and the implementation of the Packet-World.

The framework releases the developer from many difficult and error prone tasks when developing a situated multiagent system. One important example is control flow (threading) in the agent system that is fully managed by the framework. The only task to derive an application from the framework is to implement the various hot spots and use the available factories to instantiate the application. However, the framework has many hot spots. The large number of hot spots keeps the framework generic, yet the price is more work to implement the application specific parts of the application. The cookbook [34] aims to guide the developer in the development process of an application with the framework. The framework is available for download, see [1].

\section{Acknowledgements}

We are grateful to Elke Steegmans for the joint research that has contributed to the framework presented in this paper. We also would like to express our appreciation to Els Helsen and Koen Deschacht for their contribution to the development of the framework and the compilation of the framework cookbook. Finally, we thank the anonymous reviewers for the valuable feedback.

\section{References}

1. DistriNet Framework for Situated Multiagent Systems (Delta), (12/2006). http://www.cs.kuleuven.be/ danny/delta.html.

2. Multiagent system, Wikipedia, (12/2006). http://en.wikipedia.org/wiki/Multi-agent_system. 
3. Lego Mindstorms, (8/2006). http://mindstorms.lego.com/.

4. LeJOS, Lego Java Operating System for the Lego Mindstorms RCX, (8/2006). http://lejos.sourgeforce.com/.

5. J. Allen and G. Ferguson. Actions and Events in Interval Temporal Logic. Journal of Logic and Computation, Special Issue on Actions and Processes, 4:531-579, 1994.

6. K. Beck and R. Johnson. Patterns Generate Architectures. In ECOOP '94: Proceedings of the 8th European Conference on Object-Oriented Programming, Lecture Notes in Computer Science, Vol. 821, London, UK, 1994. Springer-Verlag.

7. F. Bellifemine, A. Poggi, and G. Rimassa. Jade, A FIPA-compliant Agent Framework. In 4th International Conference on Practical Application of Intelligent Agents and Multi-Agent Technology, London, UK, 1999.

8. C. Boutilier and R. I. Brafman. Partial-order planning with concurrent interacting actions. Journal on Artificial Intelligence Research, 14:105-136, 2001.

9. J. Bryson. Intelligence by Design, Principles of Modularity and Coordination for Engineering Complex Adaptive Agents. PhD Dissertation, MIT, USA, 2001.

10. L. Claesen. Regional Synchronization in Situated Multiagent Systems. Master Thesis, Katholieke Universiteit Leuven, Belgium, 2004.

11. M. Fayad and D. Schmidt. Object-Oriented Application Frameworks, Guest Editorial. Communications of the ACM, Special Issue on Object-Oriented Application Frameworks, 40(10):32-38, 1997.

12. J. Ferber. Introduction to Distributed Artificial Intelligence. Addison-Wesley, 1999.

13. J. Ferber, O. Gutknecht, and F. Michel. From Agents to Organizations: an Organizational View of Multi-Agent Systems. In Agent-Oriented Software Engineering (AOSE) IV, Lecture Notes in Computer Science, Vol. 2935. Springer-Verlag, 2004.

14. J. Ferber, F. Michel, and J. Baez. AGRE: Integrating environments with organizations. In 1st International Workschop on Environments for Multiagent Systems, Lecture Notes in Computer Science, Vol. 3374. Springer-Verlag, 2005.

15. J. Ferber and J. Muller. Influences and Reaction: a Model of Situated Multiagent Systems. 2nd International Conference on Multi-agent Systems, Japan, AAAI Press, 1996.

16. FIPA. Foundation for Intelligent Physical Agents, FIPA Abstract Architecture Specification. http://www.fipa.org/repository/bysubject.html, (8/2006).

17. N. Griffiths, M. Luck, and M. d'Iverno. Cooperative Plan Annotation through Trust. In UK Workshop on Multi-Agent Systems, Liverpool, UK, 2002.

18. O. Gutknecht, J. Ferber, and F. Michel. Integrating tools and infrastructures for generic multi-agent systems. In AGENTS '01: Proceedings of the fifth international conference on Autonomous agents, pages 441-448, New York, NY, USA, 2001. ACM Press.

19. D. Kinny, M. Ljundberg, and A. Rao. Planning with Team Activity. In 4th European Workshop on Modelling Autonomous Agents in a Multi-Agent World, Lecture Notes in Computer Science, Vol. 830. Springer-Verlag, London, UK, 1992.

20. F. Michel, A. Gouaich, and J. Ferber. Weak Interaction and Strong Interaction in Agent Based Simulations. In Multi-Agent-Based Simulation III, Lecture Notes in Computer Science, Vol. 2927. Springer-Verlag, 2003.

21. K. Rosenblatt and D. Payton. A Fine Grained Alternative to the Subsumption Architecture for Mobile Robot Control. International Joint Conference on Neural Networks, IEEE, 1989.

22. E. Steegmans, D. Weyns, T. Holvoet, and Y. Berbers. A Design Process for Adaptive Behavior of Situated Agents. In Agent-Oriented Software Engineering V, 5th International Workshop, AOSE, New York, Lecture Notes in Computer Science, Vol. 3382. Springer, 2004.

23. T. Tyrrell. Computational Mechanisms for Action Selection. PhD Dissertation, University of Edinburgh, 1993. 
24. D. Weyns, A. Helleboogh, and T. Holvoet. The Packet-World: a Test Bed for Investigating Situated Multi-Agent Systems. In Agent-based applications, platforms, and development kits. Whitestein Series in Software Agent Technology, 2005.

25. D. Weyns and T. Holvoet. Look, Talk, and Do: A Synchronization Scheme for Situated Multiagent Systems. In UK Workshop on Multi-Agent Systems, Oxford, UK, 2002.

26. D. Weyns and T. Holvoet. Model for Simultaneous Actions in Situated Multiagent Systems. In Multiagent System Technologies, Erfurt, Germany, Lecture Notes in Computer Science, Vol. 2831. Springer Verlag, 2003.

27. D. Weyns and T. Holvoet. A Colored Petri Net for Regional Synchronization in Situated Multiagent Systems. In 1st International Workshop on Coordination and Petri Nets, Bologna, Italy, 2004.

28. D. Weyns and T. Holvoet. Formal Model for Situated Multi-Agent Systems. Fundamenta Informaticae, 63(1-2):125-158, 2004.

29. D. Weyns and T. Holvoet. Regional Synchronization for Situated Multi-agent Systems. In 3th International Central and Eastern European Conference on Multi-Agent Systems, Prague, Czech Republic, Lecture Notes in Computer Science, Vol. 2691. Springer Verlag, 2004.

30. D. Weyns, K. Schelfthout, T. Holvoet, and T. Lefever. Decentralized control of E'GV transportation systems. In 4th Joint Conference on Autonomous Agents and Multiagent Systems, Industry Track, Utrecht, The Netherlands, 2005. ACM Press, New York, NY, USA.

31. D. Weyns, E. Steegmans, and T. Holvoet. Integrating Free-Flow Architectures with Role Models Based on Statecharts. In Software Engineering for Multi-Agent Systems III, Lecture Notes in Computer Science, Vol. 3390. Springer, 2004.

32. D. Weyns, E. Steegmans, and T. Holvoet. Protocol Based Communication for Situated MultiAgent Systems. In 3th Joint Conference on Autonomous Agents and Multi-Agent Systems, New York, USA, 2004. IEEE Computer Society.

33. D. Weyns, E. Steegmans, and T. Holvoet. Towards Active Perception in Situated Multi-Agent Systems. Applied Artificial Intelligence, 18(9-10):867-883, 2004.

34. D. Weyns, E. Steegmans, T. Holvoet, E. Helsen, and K. Deschacht. Delta Framework Cookbook. In Technical Report 473. Departement of Computer Science, Katholieke Universiteit Leuven, Belgium. http://www.cs.kuleuven.ac.be/publicaties/rapporten/CW/2007/, (1/2007). 